Chamorro, C., Hernández, D.,Posada, A.y Roldan, J.(2020). Formación verdeen losprogramas de contaduría pública de las universidades de Antioquia. Contaduría Universidad de Antioquia, 77, 109-129.

Doi: https://doi.org/10.17533/udea.rc.n77a04

\title{
Formación verde en los programas de contaduría pública de las universidades de Antioquia
}

Candy Lorena Chamorro González

candiilorena@gmail.com Universidad Católica Luis Amigó Orcid: 0000-0001-7332-8566

Daniela Hernández Villa daniela.hernandezvi@amigo.edu.co Universidad Católica Luis Amigó Orcid: 0000-0003-2826-3921

Angélica Posada Arias angelica.posadaar@amigo.edu.co Universidad Católica Luis Amigó Orcid: 0000-0002-9558-0970 Juliana María Roldan Vásquez juliana.roldanva@amigo.edu.co Universidad Católica Luis Amigó orcid:0000-0003-2787-3242 
Formación verde en los programas de Contaduría Pública de las universidades de Antioquia Resumen: La formación verde se convierte en una necesidad para todos los profesionales, por consiguiente, es preciso ampliar el enfoque de la enseñanza contable y abarcar conocimientos sociales y ambientales. La investigación se centró en analizar el contenido de la formación ambiental o verde en los programas de contaduría pública con respecto al contexto disciplinar actual. La metodología se fundamentó en un enfoque cuantitativo, con alcance descriptivo y método deductivo. Los resultados indican que 15 universidades de Antioquia contemplan la formación en contabilidad verde, donde el 69\% de los contenidos impartidos son de carácter interdisciplinar y el $31 \%$ son disciplinares. Se concluye que la formación ambiental tiene mayor énfasis en materia interdisciplinar, como legislación ambiental, ecología y medio ambiente, contaminación ambiental, y gestión ambiental; olvidando los contenidos disciplinares que se constituyen como un pilar fundamental del principio generativo en la formación integral del estudiante de contaduría.

Palabras clave: Contabilidad verde, formación ambiental, formación integral.

\section{Green training in Public Accounting programs at universities in Antioquia}

Abstract: Green training has become a need for all professionals, so the focus of accounting teaching should be broadened to encompass social and environmental knowledge. The research focused on analyzing the content of environmental or green training in the accounting programs regarding the current disciplinary context. The methodology was based on a quantitative approach, with a descriptive scope and deductive method. The results indicate 15 universities from Antioquia include training in green accounting, where $69 \%$ of the contents taught are of an interdisciplinary nature and $31 \%$ are disciplinary. It is concluded that environmental training has greater emphasis in interdisciplinary areas, such as environmental legislation, ecology and the environment, environmental pollution, and environmental management; while neglecting disciplinary contents which constitute a fundamental pillar of the generative principle in the comprehensive training of the accounting student.

Keywords: Green accounting, environmental training, comprehensive training.

Formação verde nos programas de Contabilidade Pública das universidades de Antioquia

Resumo: A formação verde se torna uma necessidade para todos os profissionais, por tanto, é preciso ampliar o foco do ensino contábil e abranger conhecimentos sociais e ambientais. A pesquisa centrou-se em analisar o conteúdo da formação ambiental o verde nos programas de contabilidade pública ao respeito do contexto disciplinar atual. A metodologia fundamentou-se em um enfoque quantitativo, com alcanço descritivo e método dedutivo. Os resultados mostram que 15 universidades de Antioquia contemplam a formação em contabilidade verde, onde o 69\% dos conteúdos ministrados são de índole interdisciplinar e o $31 \%$ são disciplinares. Conclui-se que a formação ambiental tem maior ênfase no interdisciplinar, como legislação ambiental, ecologia e meio ambiente, poluição ambiental, e gestão ambiental; esquecendo os conteúdos disciplinares que se constituem como um pilar fundamental do princípio generativo na formação integral do estudante de contabilidade.

Palavras chave: Contabilidade verde, formação ambiental, formação integral.

\section{La formation verte dans la Comptabilité Publique des universités en Antioquia}

Résumé: La formation verte devient une nécessité pour tous les professionnels, il est donc impératif d'élargir l'approche de l'enseignement comptable et d'y inclure les connaissances sociales et environnementales. Cette recherche s'est centrée sur l'analyse du contenu de la formation environementale ou verte dans les programmes de comptabilité publique en ce qui concerne le contexte disciplinaire actuel. La méthodologie est basée sur une approche quantitative, avec une portée descriptive, ainsi qu'une méthode déductive. Les résultats indiquent que 15 universités en Antioquia envisagent la formation en comptabilité verte, dont le $69 \%$ des contenus dispensés sont à caractère interdisciplinaire tandis que le $31 \%$ sont disciplinaires. Il est donc conclu que la formation environnementale met plus d'accent sur l'interdisciplinaire: législation environnementale, écologie et environnement, contamination environnementale, et gestion environnementale; tout en laissant de côté les contenus disciplinaires qui constituent un pilier fondamental du principe génératif dans la formation intégrale des étudiants en comptabilité.

Mots clés: Comptabilité verte, formation environnementale, formation intégrale. 
Cont. udea (julio-diciembre), pp. 109-129. (C) Universidad de Antioquia-2020.

\title{
Formación verde en los programas de contaduría pública de las universidades deAntioquia
}

\author{
Candy Lorena Chamorro González, Daniela Hernández Villa, \\ Angélica Posada Arias y Juliana María Roldan Vásquez. \\ https://doi.org/10.17533/udea.rc.n77ao4
}

Primera versión recibida en marzo de 2020 - Versión final aceptada en julio de 2020

\section{Introducción}

$\mathrm{D}$ ebido a la degradación y devastación progresiva de los recursos naturales, la humanidad viene presentando una preocupante crisis ambiental que afecta a su vez la sostenibilidad del planeta. Con ello, la formación verde se convierte en una necesidad para todos los profesionales (Reynosa, 2015). En este sentido, la disciplina contable no es indiferente ante la necesidad de incorporar fundamentos contable-ambiental en el proceso de formación, que permiten a su vez fortalecer los conocimientos para la búsqueda de soluciones respecto al deterioro del medio ambiente (Fernández y Carrara, 2009).

A partir de las diversas normatividades emitidas por el gobierno, se destacan los primeros antecedentes en materia ambiental en Colombia. A mediados del siglo XX se localiza la Ley 23 emitida en 1973 por el Congreso de la República, en donde se conceden facultades extraordinarias al presidente de la República para expedir el Código de Recursos Naturales y protección al medio ambiente y se dictan otras disposiciones, permitiéndole así al presidente de la República crear el Código Nacional de Recursos Naturales Renovables y de Protección al Medio Ambiente en el año 1974. Posteriormente, en el año 1993 el Congreso de la República emite la Ley 99 (ley general ambiental de Colombia) con el fin de crear el Ministerio del Medio Ambiente, reordenar el Sector Público encargado de la gestión y conservación del medio ambiente y los recursos naturales renovables, y organizar el Sistema Nacional Ambiental, SINA. 
Estos enlaces normativos incidieron directa e indirectamente en el desarrollo y la evolución de la contabilidad ambiental o verde en el país, puesto que se crearon normas que incidían en la aplicación de la información verde dentro del sistema contable. Entre las más importantes, se destaca la Ley 1314 del 2009, por la cual se regulan los principios y normas de contabilidad e información financiera, involucrando tres tipos de actividades especiales: actividades agrícolas, actividades de extracción y concesión de servicios.

En razón a lo precedente, el contador público como actor activo dentro de la organización, tiene la responsabilidad de velar por el cumplimiento de la normatividad ambiental y de promover dentro de las organizaciones mejores prácticas ambientales (Ablan y Méndez, 2004). Por ende, es necesario que se implementen estos conceptos desde su formación como profesional, con el propósito de brindarle elementos para la toma de decisiones que ayuden a las organizaciones a mejorar sus prácticas de producción, disminuir y aprovechar los residuos generados, y al mismo tiempo mitigar la crisis medio ambiental que se vive en la actualidad. De ahí, se vislumbra la importancia en cuanto a que las universidades contemplen dentro de su plan de estudios, teoríay conceptos de contabilidad ambiental. Mantilla citada por Rodríguez (2011) define la contabilidad ambiental como...

Un sistema que permite reconocimiento, organización, valoración y registro de las condiciones y los cambios en los recursos naturales y del ambiente, articulando indicadores de evaluación de la sostenibilidad ambiental en el contexto del desarrollo, estableciendo sistemas de información que faciliten el control y fiscalización de las acciones que afectan la condición de la naturaleza.(p.109)

La contabilidad verde ha tomado mayor importancia con el paso del tiempo, haciendo que la formación en esta área se vuelva cada vez más significativa para las universidades que imparten el pregrado de Contaduría Pública y para los contadores públicos en formación; sin embargo, algunas universidades no tienen implementado en su plan de estudios la formación ambiental o cursos electivos enfocados en estas temáticas. De igual manera, cabe destacar que muchos estudiantes perciben estos cursos como un proceso innecesario, considerándolos como "materias de relleno" y por ende tratan de evitar cursarlos.

En este sentido, cobra importancia identificar el número de universidades de Antioquia que, dentro de sus planes de estudio, contemplan la formación verde. Sin embargo, un enfoque particular de este documento se centra en analizar y reflexionar; con el fin de reconocer la formación verde o ambiental en los programas de Contaduría Pública con respecto al contexto disciplinar actual. Lo anterior permitirá inicialmente reconocer las falencias o fortalezas respecto a este tema, y además despertar la conciencia de los contadores sobre la importancia de formarse en contabilidad verde. 
Estas competencias y aprendizajes pueden contribuir al desarrollo de estrategias y herramientas que, desde la óptica contable, ayuden a perpetuar los problemas ambientales que presenta la sociedad actual. No obstante, permitirá que la formación contable se fortalezca en los enfoques ético, ambiental y social, que ayuda a la consolidación de un desarrollo económico sostenible en las organizaciones.

Finalmente, y en aporte científico, el artículo indaga sobre las discusiones discursivas acerca de la literatura ambiental que se han desarrollado; seguidamente, evidencia la importancia de incorporar la contabilidad verde en el sistema contable. Asimismo, recopila datos cuantitativos de 23 universidades de Antioquia que permiten abordar los resultados en términos de identificación y análisis en la formación ambiental o verde de los estudiantes de Contaduría Pública. En últimas, desarrolla una conclusión que contribuye a la generación de investigaciones futuras que puedan diseñar nuevas estrategias y herramientas sólidas en el campo de la investigación contable.

\section{Referente Teórico}

\section{II.I. Un abordaje literario de la Contabilidad Verde}

Las construcciones sociales y discursivas en contabilidad, proporcionan múltiples declaraciones y proclamaciones que traslumbran la necesidad y la importancia de incorporar la contabilidad verde en el sistema contable, destacando la importancia de la formación ambiental.

Según lo anterior, es significativo revelar los retos que afronta la contabilidad verde en su caminar; ante ello Correa (2017) destaca que, aunque ha crecido en popularidad, una serie de problemas con la contabilidad verde han restringido la atención de los interesados y la adopción de la gestión de las técnicas hasta el momento. Estos problemas se relacionan con los componentes fundamentales de la contabilidad verde: la contabilidad verde externa y la contabilidad de gestión ambiental (Arguello, 2018; Montero y Betancur, 2018).

La contabilidad verde externa integra la información ambiental a las partes interesadas. En este sentido, cobra importancia la identificación, clasificación y medición de los impactos ambientales en la situación económica de las empresas, ilustrados en los respectivos registros contables (Spence, Chabrak, y Pucci, 2013). Sin embargo, la disponibilidad y la calidad de las divulgaciones externas de contabilidad verde permanecen bloqueadas en los problemas del lavado verde y el lavado marrón.

Según Kim y Lyon (2015) el lavado verde es originado cuando las partes interesadas no tienen medidas directas de las emisiones de gases de los efectos invernaderos y confían en los gerentes para abordar la complejidad del modelado, los procesos de estimación y las suposiciones hechas detrás de los 
datos divulgados. Así mismo, el lavado marrón se ve alentado por el miedo a las reacciones adversas del mercado de valores y al desarrollo positivo en el desempeño ambiental, como ganar premios ecológicos, en lugar del desempeño monetario (Rout, 2010). Estos tipos de lavados se convierten en una barrera que obstaculiza la evolución de la contabilidad verde, en términos de disminución de la veracidad de los datos revelados y el escepticismo sobre la credibilidad de los informes.

Otro de los componentes que interrelaciona problemas ante el progreso de la contabilidad verde es la "contabilidad de gestión ambiental", debido a que muchas de las orientaciones afines a las decisiones de gestión se relacionan en gran medida con la falta de disponibilidad y la mala calidad de los datos, la necesidad de cambiar la información recopilada por los sistemas de control de gestión existentes y la necesidad de introducir infraestructura para recopilar y compartir información en las cadenas de suministro (Kitada y Kokubu, 2019; Álvarez, 2019). En síntesis, el problema se ubica en que los datos contables en términos de eco-eficiencia se rastrean con fines monetarios, puesto que si faltan datos de eco-eficiencia habrá menos incentivos para los gerentes que adopten la contabilidad de gestión ambiental, ya que los cálculos serán oportunidades poco confiables y rentables para los stakeholders.

Con ello, se observa que si bien existen múltiples barreras que obstaculizan el avanzar de la contabilidad verde, también se reconocen estrategias alentadoras que ayudan a presionar "el pedal de la aceleración" en el desarrollo de procesos y actividades alineados a la contabilidad, economía, equidad social y medio ambiente.

Consecuentemente, se considera que el surgimiento de la Industria 4.o, podría proporcionar una base para superar estos desafíos ubicados tanto en la contabilidad verde externa, como en la contabilidad de gestión ambiental. Burritt y Christ (2016) expresan que la mejora en la tecnología produce una digitalización extensa que co-ayuda a (1)generar calidad en los datos contables ambientales reportados, en términos de oportunidad, precisión, confiabilidad y comparabilidad, (2) reduce el lavado verde y marrón, (3) mitiga la discreción de gestión sobre lo que se mide y cómo se mide e informa. Macias y Velásquez (2017) Sommer (2015) destacan que estos impulsos permitirán cumplir con los imperativos ambientales del futuro, que se requiere una información más rica.

A partir de los argumentos mencionados, cobra importancia definir el término de contabilidad verde, que de acuerdo con Ochoa, Mosquera, y Ruiz. (2013) es "un área de la contabilidad encargada de analizar los procesos de medición, valoración y control de los recursos naturales, con el fin de contribuir en la construcción de conceptos que amplíen su campo de acción en la contabilidad empresarial" (p, 14). No obstante, Hernández, citado por Chamorro (2015), define la contabilidad verde como 
...un subsistema del conocimiento contable que procura dar cuenta de la realidad ambiental, es decir, que mide, valora y controla las relaciones complejas tanto de tipo natural como social que interactúan dinámicamente en un espacio-tiempo para hacer posible la vida. $(\mathrm{p}, 56)$

En contexto, la contabilidad debe encargarse de medir, valorar, cuantificar e informar sobre los aspectos cualitativos y cuantitativos que pueden influir en la toma de decisiones acerca de las actuaciones sobre las variables medioambientales. De acuerdo con lo anterior, surge la necesidad de incorporar partidas ambientales dentro del sistema contable con el propósito de registrar la información verde en la que incurren las organizaciones, tales como activos, pasivos, ingresos, gastos, etc.

El Instituto de Contabilidad y Auditoría de Cuentas ICAC (2002), define como activo ambiental, todos aquellos elementos incorporados al patrimonio de la entidad con el objeto de ser utilizados de forma duradera en su actividad, cuya finalidad principal sea la minimización del impacto medioambiente y la protección y mejora del medioambiente, incluyendo la reducción o eliminación de la contaminación futura de las operaciones de la entidad. Dentro del grupo de activos ambientales se enmarcan los materiales que se utilicen en los procesos productivos minimizando la emisión de residuos, la propiedad, planta y equipo que se utilicen con el fin del cuidado ambiental, y los cargos diferidos representados por gastos de investigación y desarrollo en la tecnología limpia, así como los gastos de reforestación (Ablan y Méndez, 2004).

Del mismo modo, los pasivos ambiental son todas aquellas obligaciones del ente económico que deberán ser pagadas en el presente o en el futuro, las cuales han sido el resultado de actuaciones donde la organización ha participado directa o indirectamente por acción u omisión, voluntaria o involuntariamente, no cumpliendo la función de acumular, generar, distribuir y mantener los activos ambientales y las riquezas ambientales, obteniendo como resultado disminuciones en la calidad y/o cantidad de un activo ambiental o de dicha riqueza, (Mejía, Montes y Mora, 2010).

Otra de las partidas ambientales que conforman la contabilidad son los ingresos, los cuales se definen como el incremento de la calidad y/o cantidad de los activos ambientales producidos en un periodo determinado, representados en la entrada de nuevos activos ambientales o el mejoramiento de la calidad de los existentes; dichas entradas o incrementos, son derivadas de todas aquellas acciones u omisiones del hombre, o en consecuencia de la dinámica autónoma propia de la naturaleza y su interacción en el medio (Mejía y Vargas, 2012).

La contabilidad ambiental se enfoca en las unidades físicas, la financiera gira en torno a lo monetario; Bischhoffshausen (1996, citado por Mejía et al., 2010) señala que son ingresos ambientales: el ahorro de materiales y materias primas por reducción de desechos, ingresos por venta o ahorro por reutilización 
de material reciclado, ahorro de gastos de disposición final de desechos por disminución de su volumen, venta de derechos de emisión transables, ingresos por licencias de tecnologías limpias, y ahorro por sustitución de material contaminante por otro no contaminante.

Dentro de este contexto, los gastos ambientales se refieren al decremento de la calidad y/o cantidad de activos ambientales en un periodo de tiempo, representados en la salida de activos ambientales o el deterioro, pérdida o agotamiento de la calidad de los activos ambientales existentes; dichas disminuciones son el producto de las acciones u omisiones del hombre, o consecuencia de la dinámica autónoma propia de la naturaleza y su interacción en el medio (Mejía y Vargas, 2012).

En síntesis, se menciona que las partidas ambientales como activos, pasivos, ingresos y gastos, permiten integrar la información verde en cuentas financieras. Esto contribuirá a la trazabilidad de la información y la cuantificación de la contabilidad verde o ambiental. Consolidándose, además, en un primer paso para el desarrollo sostenible de las empresas que se están moviendo hacia una producción más limpia en su actividad operativa, comercial y de servicios.

\section{Formación verde en Contaduría Publica}

Desde el enfoque internacional, los investigadores expresan de manera constante la necesidad de integrar los elementos de la contabilidad verde en la formación del pregrado de Contaduría Pública (Burritt y Christ, 2016; Deegan, 2017; Van, Chamorro, Hens y Lan, 2016). En este sentido, se reconoce que el acelerado crecimiento económico ha provocado diversas transformaciones y cambios permanentes, que modifican las relaciones socio-culturales y socioambientales del entorno en que vivimos.

La globalización, los avances tecnológicos, y la apertura económica, han generado grandes beneficios para la sociedad, pero a su vez ocasionan graves daños en el medio ambiente, por ende, las tendencias enfocadas en el cuidado, conservación y protección de los recursos naturales renovables y en especial de los no renovables han tomado más relevancia con el paso del tiempo. Lo precedente ha generado que las organizaciones y demás entes económicos busquen el método para realizar sus operaciones de una forma congruente, verídica, fiable, y oportuna, que impulse la permanencia y el crecimiento económico de la organización y a la vez proteja el medio ambiente.

Ante ello, se demuestra que el contador no puede ser ajeno a estos cambios que ha de afrontar la sociedad, y en consecuencia, para cumplir con las necesidades del entorno y de las organizaciones, se requiere que los profesionales contables estén formados no solo en el campo económicofinanciero, sino también en el campo socio-ambiental. La contabilidad enfocada 
solo en el ámbito económico y financiero, no da respuestas a las demandas de información socio-ambiental que han tomado fuerza en la sociedad, limitando así las capacidades y el campo laboral de los futuros profesionales (Gómez, 2020). Por consiguiente, es preciso ampliar el enfoque de la enseñanza contable y abarcar conocimientos sociales y ambientales, los cuales son necesarios para queel profesional actué con idoneidad y pueda satisfacer las necesidades delos diferentes usuarios de la información y de la sociedad en general (Fernándezy Carrara, 2009).

En relación con lo anterior, Duque (2012) considera que la contabilidad se debe empezar a entender y enseñar aspectos que conduzcan, no solo como un mecanismo de control financiero y contable, sino también como una disciplina social capaz de generar cambio, de transformar, de buscar el bienestar común y la solución a diversas problemáticas sociales; para ello, se deben propiciar iniciativas dirigidas hacia la investigación que tengan un enfoque social y estas deben estar estrechamente relacionadas con la construcción de una mejor educación.

Desde otro punto de vista, un gran número de empresas utilizan recursos naturales para emplearlos en la producción de bienes y servicios, y parte de estos recursos, luego de ser usados, son devueltos al entorno en forma de residuos sin ningún tipo de tratamiento, provocando altos niveles de contaminación y degradación del ambiente (Arguello, 2018). Esta situación ha concientizado a la sociedad, logrando que se plantee un desarrollo sostenible que promueva el uso eficiente y la preservación de los recursos disponibles a través de las herramientas y metodologías que ofrece la disciplina contable.

Por lotanto, las empresas se deben considerar como una unidad económica que actúa en y para la sociedad, afrontando las consecuencias de la realización de sus actividades económicas. Se concibe entonces, la necesidad de romper el esquema clásico en el que las empresas buscan solo el beneficio económico y lucrativo de sus propietarios, pues además deben buscar mejores formas de operar y de consumir los recursos naturales, que permita a su vez la sostenibilidad en términos de gestión ambiental.

Ahora bien, para romper con estos esquemas desde el ámbito contable, es preciso reconocer el grado de relación que tienen el ambiente y la contaduría, capacitando a los contadores no solo en la parte financiera, sino también en la parte no financiera, donde se destaque lo social y lo ambiental como medio para generar rentabilidad sosteniblemente. En este orden de ideas, el contador público debe aprender las técnicas y procedimientos adecuados para cuantificar y revelar información, de tal forma que puede llevar un control adecuado de los recursos naturales, su asignación y uso, así como relacionar la información con los costos por el daño ambiental, evaluar y dar un tratamiento a los mismos, de manera que permita reflejar en los estados 
financieros el impacto en el medio ambiente causado por las actividades que realiza la empresa (Ablan y Méndez, 2004).

Del mismo modo, Cubides (2017) plantea que el objetivo de la contabilidad ambiental o verde es el de evaluar mediantelaidentificación, medición y costeo del entorno donde interviene, si las organizaciones con la realización de su objeto social están beneficiando o perjudicando al medio ambiente; lo cual permitirá tomar acciones preventivas o correctivas tendientes a la recuperación o mitigación del impacto ambiental causado o por causar, de manera que las organizaciones eviten sanciones por el incumplimiento de la normatividad ambiental vigente y obtengan beneficios económicos al reducir sus costos y gastos. Teniendo como resultado el reconocimiento de la restauración y cuidado con el medio ambiente, además de registrar dentro de sus operaciones las afectaciones o posibles afectaciones, orientando a una gestión organizacional más eficiente y responsable con el medio ambiente.

En este contexto, Espinoza (2017) percibe la educación como un instrumento fundamental para el crecimiento y cambio de la sociedad, por tanto, los contadores públicos deben aprender lo concerniente a la protección y manejo eficiente de los recursos naturales y la importancia de dicho impacto en las empresas, considerando así a la contabilidad verde como aquel método de asumir de una forma cuantitativa, registrada y ordenada el impacto de las afectaciones al medio ambiente, originadas por las operaciones de las organizaciones.

En contexto, la formación verde es de suma importancia para el pregrado de Contaduría Pública, pues día a día la comunidad se vuelve más consciente de los daños que se causan en el planeta por la desesperada búsqueda de desarrollo; por esto es de vital importancia que desde la academia se dicten asignaturas con énfasis en el buen uso del ambiente. En el caso puntual, la contabilidad ambiental o verde les brinda a los profesionales una amplia cantidad de herramientas, las cuales les permiten desarrollar proyectos que busquen un crecimiento en los dos frentes: (1) la permanencia y evolución económica de la empresa, (2) la protección y el uso adecuado de los recursos naturales. Con ello, se podría fortalecer ampliamente el margen de ganancia en las compañías y la calidad de vida de muchas personas, además de brindarles una enseñanza contable a los profesionales con un enfoque ético, ambiental y social, que permita un desarrollo económico sostenible.

\section{Metodología}

El presente documento se desarrolla en el campo contable - ambiental, específicamente en la formación verde de los programas de Contaduría Pública de las universidades del departamento de Antioquia. La investigación posee un enfoque cuantitativo con un alcance descriptivo. La primera 
etapa de la investigación recopila los datos de las distintas universidades que imparten el pregrado de Contaduría Pública en el departamento de Antioquia. Posteriormente se identifican las horas designadas en cada programa académico para la formación en contabilidad verde. Finalmente, se analiza el enfoque de la asignatura a través de los documentos internos de las instituciones con el fin de examinar el desarrollo y el objetivo del curso.

La población del estudio estuvo conformada por 44 universidades que se encuentran ubicadas en el departamento de Antioquia y una muestra de 23 instituciones, tanto de carácter oficial como privado, que ofrecen el programa de Contaduría Pública, en sus diferentes modalidades, presencial, a distancia o virtual.

La recopilación de la información se abordó iinicialmente a través fuentes primarias, sustentadas en artículos, libros, trabajos de grado y normatividad legal relacionada con la contabilidad ambiental o verde. No obstante, se implementaron fuentes secundarias como el sistema nacional de información de la educación superior (SNIES), los planes de estudio del pregrado de Contaduría Públicaylas cartas descriptivas de las asignaturas contabilidad ambiental o social.

El análisis de la información se desarrolló a través de la herramienta de Excel y el programa estadístico SSPS que buscaba: (1) identificar el número de universidades que incorporan el pregrado de Contaduría Pública, (2) determinar la ubicación de las universidades (3) reconocer el tipo de razón social de las instituciones según el Ministerio de Educación (4) examinar la intensidad horaria de la asignatura (3) analizar los enfoques disciplinares e interdisciplinares de la correspondiente asignatura.

\section{Resultados}

El objetivo principal de esta sección es destacar las estadísticas relacionadas con la temática de estudio en el ámbito de la profesión contable en Colombia. Los resultados, permiten analizar la importancia de la formación ambiental en la disciplina contable y cómo esta puede contribuir a perpetuar los problemas verdes a través de los profesionales y las entidades, quienes son las primeras responsables en la ejecución de acción que entrelacen la gestión y la contabilidad ambiental o verde.

En Colombia existen actualmente 139 universidades, de estas 44 Universidades se encuentran ubicadas en el departamento de Antioquia, pero solo 23 instituciones tanto de carácter oficial como privado ofrecen el pregrado de Contaduría Pública, en sus diferentes modalidades, presencial, a distancia y virtual; muchas de estas cuentan con sedes en las diferentes subregiones del departamento; sin embargo, no todas consideran, dentro de la formación profesional del contador público, la formación ambiental. De acuerdo con la 
Figura 1, se reconoce que únicamente 15 de estas universidades contemplan dentro de su plan de estudios la formación verde de sus estudiantes.

Figura 1: Cantidad de Universidades en el departamento de Antioquia

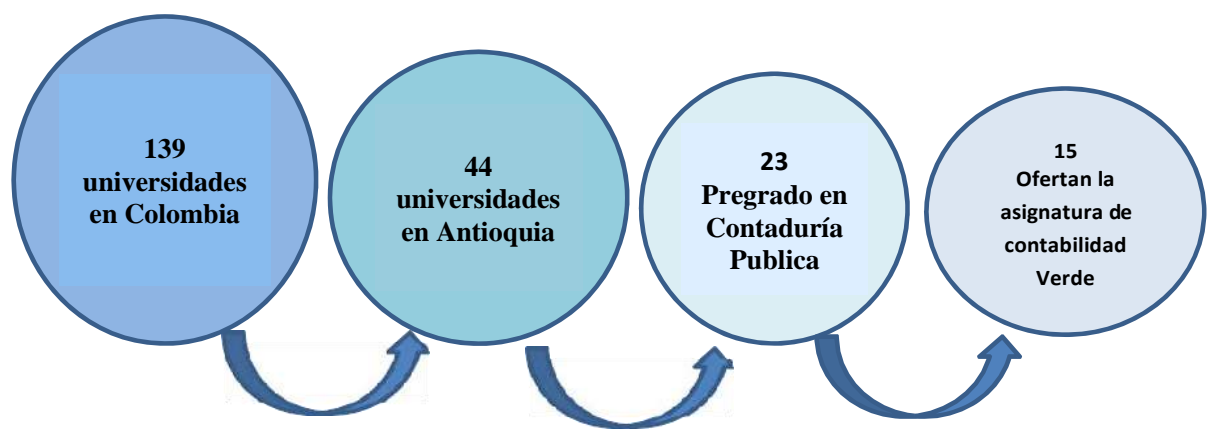

Fuente: Elaboración propia (2019).

Este hallazgo toma en consideración los argumentos afirmados por Papagiannakis, Voudouris, Lioukas y Kassinis (2019) quienes manifiestan que las organizaciones requieren profesionales contables con valor agregado en materia ambiental, puesto que actualmente se evidencian distintos estándares que demandan medir y evaluar activos, pasivos, ingresos, costos y gastos ambientales; sin embargo, existen vacíos educativos en los contadores públicos en materia ambiental debido a que gran parte de los programas de Contaduría Pública no contienen una asignatura enfocada en contabilidad ambiental (Hens et al., 2018). Por consiguiente, las universidades de Colombia deben sumar esfuerzos internos que propendan por la incorporación de asignaturas de contabilidad verde.

De igual manera es importante resaltar los lineamientos de Bui, et al.(2017) en relación con que los profesionales contables también son ciudadanos que tienen la responsabilidad de gestionar acciones y procesos que conduzcan a un crecimiento resiliente y a la reducción de la vulnerabilidad frente a los riesgos de desastres y al cambio climático que desarrollan las empresas. Lo anterior condiciona a los programas de Contaduría Pública a gestionar mecanismos de evaluación que ayuden alas empresasya los profesionales en la implementación de sistemas o herramientas en pro del medio ambiente, como lo es la contabilidad ambiental o verde. 
Figura 2: Ubicación y tipos de instituciones en educación verde
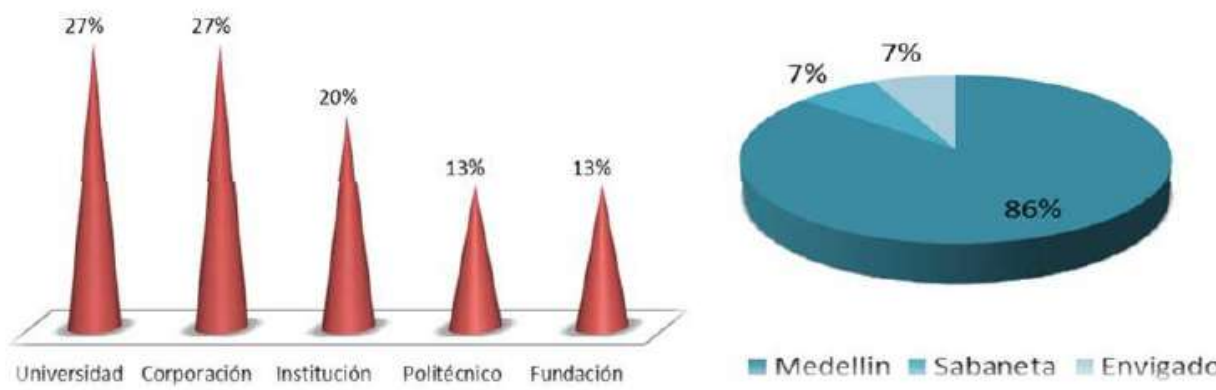

Fuente: Elaboración propia (2019).

Ahora bien, las universidades que incluyen este tipo de formación, se encuentran principalmente en la capital del departamento, con un 86\%, seguidamente se ubica una igualdad porcentual entre los municipios de envigado y sabaneta con un $14 \%$. Lo anterior, identifica que en la ciudad de Medellín han contemplado con "responsabilidad" la necesidad de formar a sus estudiantes en contabilidad y ambiente, ayudando a fortalecer las competencias que debe integrar un profesional de Contaduría Pública.

Adicionalmente, la figura 2 ilustra los tipos de instituciones que incluyen una educación verde en los programas de Contaduría Pública. Logrando identificar que gran parte de las instituciones educativas mantiene en su razón social "universidad" y "corporación”, en ese orden se ubican las "instituciones" en último lugar y con un puntaje igual se hallan Politécnico y Fundación. Situación que permite destacar información demográfica en la investigación.

Paralelamente, es de suma importancia que los estudiantes de Contaduría Pública reciban una formación integral en los aspectos ambientales y sociales; para lograr esto, es oportuno que estas universidades impartan este tipo de contenidos con una intensidad de 4 horas semanales (64 horas semestrales), como sucede con otras asignaturas de igual relevancia como lo son la tributaria o los costos (González, 2015). 
Chamorro,C., Hernández,D.,Posada,A.yRoldan,J.Formaciónverdeenlosprogramasdecontaduría...

Figura 3: Intensidad horaria de la formación

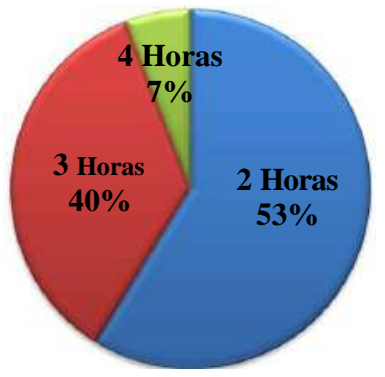

Fuente: Elaboración propia (2019).

La información recolectada permitió identificar que de un total de 15 Universidades, solo la Universidad Autónoma Latinoamericana, correspondiente al $7 \%$, dicta 4 horas semanales, considerado como el tiempo adecuado y necesario para tener una formación verde completa. Paralelamente, se identificó que la Corporación Universitaria Americana, la Universidad EAFIT, el Politécnico Colombiano Jaime Isaza Cadavid, la Universidad Católica Luis Amigó, la Fundación Universitaria María Cano, y el Politécnico Grancolombiano, que equivalen al 40\%, tienen una intensidad de 3 horas semanales (48 horas semestrales). Finalmente, se reconoce que la Corporación Universitaria Remington, Instituto Tecnológico Metropolitano, Fundación Escuela Colombiana de Mercadotecnia, Corporación Universitaria de Sabaneta, Corporación Universitaria U de Colombia, Universidad de San Buenaventura, la Institución Universitaria Salazar y Herrera y la Universidad de Envigado, que conforman el 53\% restante, brindan una formación ambiental de 2 horas a la semana (32 horas en el semestre), (ver Figura 3).

En este sentido, se analiza que, aunque estas universidades estén formando a sus estudiantes de Contaduría Pública en contenidos socio-ambientales, las horas dictadas no son suficientes para abarcar todos los temas de esta asignatura y formar profesionales con una conciencia socio-ambiental que les permita en un futuro tener las herramientas y las capacidades para proponer soluciones y afrontar las necesidades que las empresas y la sociedad tengan respecto a estos temas.

En consecuencia, es por ello que muchos profesionales de Contaduría Pública presentan vacíos conceptuales en contabilidad verde y se ven obligados a realizar seminarios o cursos alternos para formarse en esta especialidad, puesto que son conscientes de que estos saberes son un conocimiento integral que necesita con urgencia el mundo empresarial e investigativo. Esta situación da un retroceso al avance de este tipo de contabilidad, debido a que 
los contadores públicos son la figura principal para vigilar y controlar que la aplicación de la contabilidad verde en las organizaciones (Evangelinos, Nikolaou y Leal, 2015).

Así mismo, los resultados se interrelacionan con los planteamientos emitidos por Novillo y Hachi (2014) e Higuera (2015), quienes plantean la necesidad de diseñar propuestas metodológicas amplias para la formación de los estudiantes de Contaduría Pública en contabilidad ambiental con el objetivo de impartir y formar todas las temáticas que se vinculan al eje epistemológico y práctico de la contabilidad verde. Esta herramienta pedagógica permitirá establecer la intensidad horaria necesaria en el proceso de enseñanza y aprendizaje en contabilidad verde (Urraca y Silva, 2017). Lo anterior ayudará a fortalecer las competencias de los contables y, así mismo, incrementar el desarrollo humano y social de las organizaciones que contrarrestan la problemática ambiental que hasta hoy ha sido irrefrenable.

Ahora bien, la figura 4 da cuenta de los aspectos que destacan la profundidad de la formación en la asignatura de contabilidad verde. Para ello, fue necesario dividir la formación desde dos ópticas (1) eje disciplinar y (2) eje interdisciplinar, con el objetivo de visualizar el enfoque que prevalece en el aprendizaje de contabilidad verde.

De acuerdo a ello, se logró establecer que, de 15 universidades, el foco disciplinar se encuentra distribuido en un $11 \%$ para la normatividad de la contabilidad ambiental, el 6\% para la definición de partidas ambientales y el concepto de contabilidad ambiental, un $3 \%$ para los aspectos de tributación y sistema contables, finalmente el $1 \%$ es enfocado en modelos de contabilidad verde y responsabilidad socio-ambiental del contador público.

Figura 4: Profundización de la formación ambiental

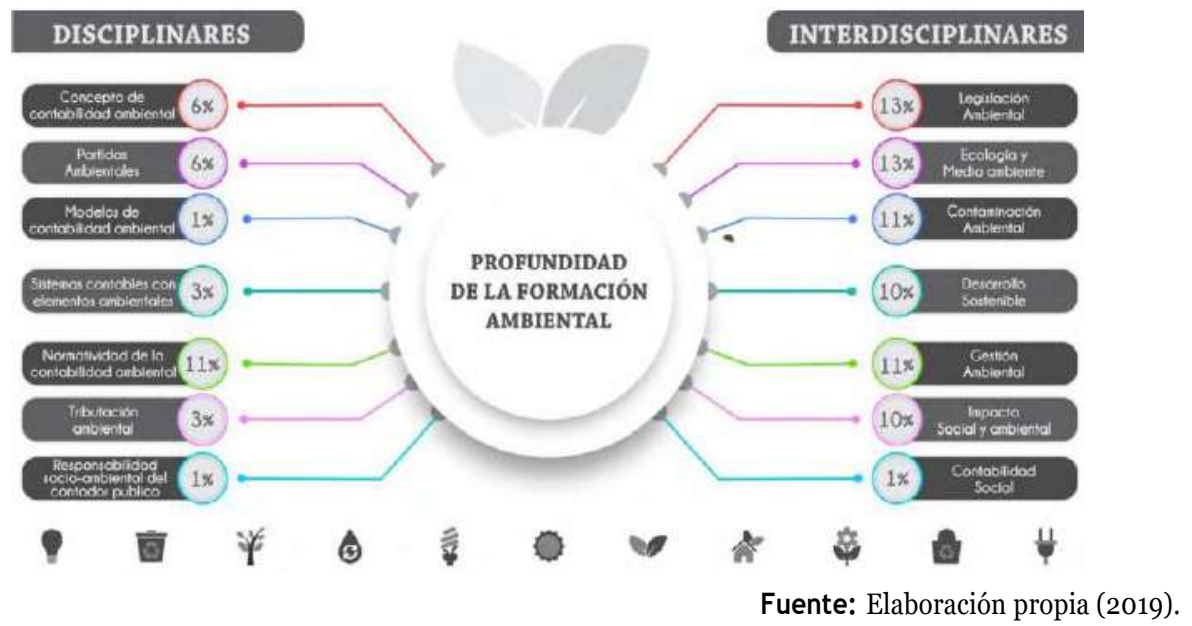


Bajo la misma óptica, se analizó el área interdisciplinar dela asignatura que se encuentra liderada por un 13\% en el aprendizaje de legislación ambiental y conceptos de ecología o medio ambiental; paralelamente se ubican con un 11\% temas de gestión ambiental y contaminación; un 10\% de la asignatura corresponde al perfeccionamiento de aspectos en temas de desarrollo sostenible e impacto social; finalmente ubican el aprendizaje de contabilidad social en un $1 \%$.

Estos hallazgos concuerdan con las expresiones de Taleb (2015), quien observó en su estudió que las pocas asignaturas relacionadas con contabilidad ambiental están construidas desde contenidos interdisciplinares y han evidenciado algunas dificultades con la coherencia horizontal en las teorías que sustenta la contabilidad ambiental. Así mismo, persiste una inexistencia con la incorporación de los proyectos trasversales obligatorios e institucionales, el fomento de la investigación y la pertinencia con el contexto ambiental actual.

De acuerdo con los datos analizados, se considera que una de las principales tareas es el desarrollo depropuesta de enseñanzay aprendizajeen contabilidad verde, que permitan trazar estrategias de mejora y solución para las dificultades evidenciadas. Es así como Welford (2016) establece que los planes educativos en educación verde deben estar alineados desde la óptica constructivista que permita una educación que dé mayor importancia al contexto de aprendizaje que a la memorización. Esto permitirá fortalecer las habilidades, capacidades y las competencias de los estudiantes contables en materia ambiental (Wójcik, 2015).

En este sentido, los hallazgos permiten analizar la necesidad de fortalecer el eje disciplinar de la contabilidad verde. Estos conceptos les permitirán a los profesionales contables desarrollar estrategias de sensibilización ambiental, evaluar los sistemas productivos ambientales de las organizaciones y reconocer dentro de los informes financieros las cuentas socio- ambientales que a su vez ayudan a configurar procesos e inversiones responsables que han de ejecutar para el desarrollo humano y social de la entidad. Nos obstante contribuirá a que las organizaciones desarrollen mejoras en las cadenas de suministro y operaciones en respuesta a la adaptación del cambio climático y el aumento de la resiliencia de las comunidades ante la recuperación y el mantenimiento del ecosistema (Lieder y Rashid, 2016).

Tomando todos los elementos en consideración, la presente investigación reconoce que los programas de Contaduría Pública estudiados priorizan el eje interdisciplinar. Por tanto, es axiomático que estos programas incorporen lineamientos o estrategias educativas que permitan repensar la contabilidad verde adherida a los componentes disciplinares. Logrando así conocer las teorías que sustentan la contabilidad verde desde la corriente funcionalista y heterodoxa. Esto es absolutamente necesario para confrontar la lógica de la 
contabilidad verde y construir nuevos criterios de ubicación conceptual que permitan dinamizar estrategias de representación social y ambiental.

\section{Conclusiones}

La investigación abordada gira en torno a la necesidad de implementar la educación contable-ambiental en las universidades del departamento de Antioquia, esto con base en la preocupación existente por la poca conciencia social que tiene el hombre por el cuidado del medio ambiente y cómo desde la formación contable se pueden generar cambios importantes para la sociedad, incrementando la consciencia sobre los daños que día a día esta le causa al planeta en la búsqueda desesperada del desarrollo.

De acuerdo con ello, el estudio reveló la importancia de la formación en materia ambiental dentro de los programas de Contaduría Pública impartidos en las universidades del departamento de Antioquia, ya que la contabilidad además de servir a las organizaciones como una herramienta de control financieroy gestión, también deberá evidenciar el uso que estas le están dando a los recursos naturales, controlando toda acción que afecte la condición de la naturaleza y el desarrollo sostenible.

A su vez, la contabilidad debe ser vista no solo como un mecanismo de control financiero y contable, sino también, como una disciplina social generadora de cambio y transformación, que busca el bienestar común y la solución a diversas problemáticas sociales, por lo que se deben tomar iniciativas dirigidas hacia la investigación, las cuales contengan un enfoque social generando, así mismo, la construcción de una mejor educación.

Tomando en cuenta los resultados obtenidos, se concluye que dentro del departamento de Antioquia se ubican 23 universidades que ofrecen el pregrado de Contaduría Pública y 15 de estas contemplan el curso de contabilidad ambiental dentro de su malla curricular. Analizando así que el $65 \%$ de las universidades ubicadas en Antioquia forman a sus estudiantes en diferentes principios y postulados que existen en contabilidad verde. Lo anterior genera un progreso positivo en el eje problemático de la investigación. Sin embargo, aún es necesario que las universidades promuevan la formación ambiental en los estudiantes de Contaduría Pública desde un enfoque disciplinario e interdisciplinario.

Por otro lado, se menciona que las universidades direccionan con un gran volumen porcentual la formación ambiental desde el enfoque interdisciplinario, análisis que se fundamenta a partir de los 15 planes de estudios revisados, en donde se halló que los aspectos interdisciplinarios equivalen al 69\%, mientras que los disciplinarios al $31 \%$.

En este contexto, se reflexiona que, aunque existe un progreso en la formación ambiental de los futuros profesionales de Antioquia, se reconoce que 
este aprendizaje no está direccionado desde la óptica disciplinar, situación que genera un reproceso en las competencias que deberá adquirir el estudiante para implementar, manejar y ejecutar la contabilidad verde en una organización, que a su vez ayuda a perpetuar los problemas ambientales que presenta el planeta.

Por tanto, se determina la importancia de abordar la temática de la contabilidad verde desde las corrientes principales de la contabilidad que dan cuenta de las teorías, modelos, herramientas, instrumentos y otros aspectos que vislumbran el corpus del conocimiento en relación a los principios y características que se vinculan a la contabilidad verde. Con ello los contadores ayudarán a que las organizaciones puedan tomar medidas que promuevan mejoras en las prácticas ambientales y el buen uso de los recursos naturales.

Así mismo, los profesionales podrán analizar que la contabilidad tiene como objetivo simplificar lo complejo, pero en ello no solo se ubica lo instrumental sino también la sociedad; reflejando así la preocupación por el ambiente que re direcciona a elementos como la vida, la salud, la educación, la cultura, entre otros.

Finalmente, se recomienda que las investigaciones futuras repliquen este foco de investigaciones en otros departamentos de Colombia, a fin de contribuir en el despertar de la conciencia de los programas de Contaduría Pública y en los mismos profesionales, en cuanto a la formación en temas verdes o ambientales se refiere.

No obstante, se cree que la reflexividad es una manera de fomentar el compromiso de los académicos con la práctica de la contabilidad verde, un medio para mejorar la investigación y transformar las barreras de dominación que permitan el desarrollo de nuevos principios y postulados y, a su vez, complementen las teorías y los modelos existentes.

\section{Referencias bibliográficas}

Ablan, N., y Méndez, E. (2004). Contabilidad y Ambiente. Una disciplina y un campo para el conocimiento y la acción. Actualidad contable faces, 7(8), 7-22.

Álvarez, H. (2019). Buscando la Ruta dela medición-valoración ecológica no monetaria, en la teoría tridimensional de la contabilidad. Visión contable. 20, 22-95.

Arguello, M. (2018). La problemática de la Contabilidad Ambiental. Revista Publicando, 5(15 (2), 199-208.

Bui, N., Kawamura, A., Kim, K., Prathumratana, L., Kim, T., Yoon, S., y Truong, N. (2017). Proposal of an indicator-based sustainability assessment framework for the mining sector of APEC economies. Resources Policy, 52 (405-417). http://doi.org/10.1016/j. resourpol.2017.05.005

Burritt, R., \& Christ, K. (2016). Industry 4.0 and environmental accounting: a new revolution?.Asian Journal of Sustainability and Social Responsibility, 1(1), 23-38.

Chamorro, C. (2015). Estado actual de la contabilidad verde en Colombia. Saber, ciencia y libertad, $10(2), 53-62$. 
Congreso de la Republica de Colombia. (13 de Julio de 2009). Ley 1314. Por la cual se regulan los principios y normas de contabilidad einformación financiera y de aseguramiento de información aceptados en Colombia. Diario Oficial No. 47.409.

Congreso de la Republica de Colombia. (19 de Diciembre de 1973). Ley 23. Por el cual se conceden facultades extraordinarias al Presidente de la República para expedir el Código de Recursos Naturales y de Protección al Medio Ambiente y se dictan otras disposiciones. Diario Oficial No. 34.001.

Congreso de la Republica de Colombia. (22 de Diciembre de 1993). Ley 99. Por la cual se crea el Ministerio del Medio Ambiente, se reordena el Sector Público encargado de la gestión y conservación del medio ambiente y los recursos naturales renovables, se organiza el Sistema Nacional Ambiental, y se dictan otras disposiciones. Diario Oficial No. 41.146.

Correa,D.(2017).Sistema detareas docentesparaintroducirladimensión ambientalenladisciplina Contabilidad de la carrera de Contabilidad y Finanzas. (Bachelor's thesis). Universidad de Holguín, Facultad de Ciencias Económicas y Administración, Departamento de Contabilidad y Finanzas. Cuba.

Cubides, A. (2017). Importancia y aplicabilidad de la Contabilidad Ambiental en las empresas colombianas. Bogotá D.C. Colombia: Universidad Militar Nueva Granada.

Deegan, C. (2017). Twenty five years of social and environmental accounting research within Critical Perspectives of Accounting: Hits, misses and ways forward. Critical Perspectives on Accounting, 43, 65-87.

Duque, F.(2012). Una mirada crítica a la formación del contador público en Colombia. Adversia (11), 1-11.

Espinoza, Z. (2017). Contabilidad Ambiental como Herramienta de Responsabilidad Social. Lima: Universidad Señor de Sipán Pimentel.

Evangelinos, K., Nikolaou, I., \& Leal Filho, W. (2015). The effects of climate change policy on the business community: a corporate environmental accounting perspective. Corporate Social Responsibility and Environmental Management, 22(5), 257-270.

Fernández, L. y Carrara, C. (2009). Enfoque socio-ambiental en la formación del contador público. Visión de futuro, 12(2), 1-15.

Fundación Fórum Ambiental.(1999). Contabilidad ambiental: medida, evaluación y comunicación de la actuación ambiental de la empresa. Recuperado de: http://www.forumambiental. org/es/contabilidad-ambiental-medida-evaluacion-y-comunicacion-de-la-actuacionambiental-de-la-empresa2/

Gómez, J. (2020). Contabilidad, paradigma de los conjuntos: Etimología, historia y perspectivas futuras. Alternativa Financiera, 9(1). 25-38.

Hens, L., Block, C., Cabello-Eras, J., Sagastume-Gutierez, A., Garcia-Lorenzo, D., Chamorro, C., \& Vandecasteele, C. (2018). On the evolution of "Cleaner Production" as a concept and a practice. Journal of Cleaner Production, 172, 3323-3333.

Higuera, E. (2015). Valoración del costo ambiental estimado en los EEFF de la Universidad Militar Nueva Granada sede Bogotá. (Bachelor’s thesis). Universidad Militar Nueva Granada. Colombia.

Kim, E. y Lyon, T. (2015). Greenwash vs. brownwash: exageración y modestia indebida en la divulgación de sostenibilidad corporativa. Organization Science, 26(3), 705-723. 
Chamorro,C., Hernández,D.,Posada,A.yRoldan,J.Formaciónverdeenlosprogramasdecontaduría...

Kitada, H. y Kokubu, K. (2019). El papel de los indicadores en la integración del proceso de sostenibilidad en las actividades corporativas: estudios de casos de empresas japonesas. Gestión dela sostenibilidadyestrategia empresarialenAsia,16, 1.

Lieder, M., \& Rashid, A. (2016). Towards circular economy implementation: A comprehensive review in context of manufacturing industry. Journal of Cleaner Production. 115, 36-51. http://doi.org/10.1016/j.jclepro.2015.12.042

Macías, H. y Velásquez, S. (2017). Avances de la contabilidad social y ambiental en su contexto original. Contaduría Universidad de Antioquia, 70, 13-41.

Mejía, E., y Vargas, L. (2012). Contabilidad para la sostenibilidad ambiental y social. Lumina, 13, 48-70.

Mejía Soto, E., Montes Salazar, C., y Mora Roa, G. (2010). Estructura conceptual de la Teoría Tridimensional de la Contabilidad. Contexto, 2, 34-53.

Montero, M. y Betancur, E. (2018). Una mirada a la contabilidad ambiental. Postulados y retos. Contaduría Universidad de Antioquia, (73), 199-209.

Novillo, M. \& Hachi, J. (2014). Metodología para contabilizar los aspectos ambientales generados por instituciones públicas y privadas en base al cumplimiento de la normativa ambiental vigente. (Master's thesis). Universidad Politécnica Salesiana. Ecuador.

Ochoa, C., Mosquera, C., y Ruiz, C. (2013). Modelo de contabilidad ambiental para la empresa Pedro Álvarez construcciones S.A.S. (Trabajo de grado). Medellín, Colombia. Recuperado de: http://bibliotecadigital.usb.edu.co/handle/10819/1619

Papagiannakis, G., Voudouris, I., Lioukas, S. y Kassinis, G. (2019). Sistemas de gestión ambiental e innovación de productos ambientales: el papel de la participación de los interesados. Estrategia empresarialy medio ambiente, 28(6), 939-950.

Presidencia de la Republica de Colombia. (18 de Diciembre de 1974). Decreto 2811. Por el cual se dicta el Código Nacional de Recursos Naturales Renovables y de Protección al Medio Ambiente. Diario Oficial No 34.243.

Reynosa, E. (2015). Crisis ambiental global: Causas, consecuencias y soluciones prácticas. Recuperado de Acta Académia: https://www.aacademica.org/ern/5.pdf

Rodríguez,D.(2011). Formulación de una estructura teórica para la contabilidad ambiental. Libre empresa, 8(2), 101-120.

Rout, H. (2010). Green Accounting: Issues and Challenges. Journal of Managerial Economics. Aug. 8(3), 46-60.

Sanz, C. (1995). El medio ambiente y la contabilidad. Acciones e investigaciones sociales (3), $165-178$.

Sommer, L. (2015). Revolución industrial-industria 4.0: ¿son las PYME manufactureras alemanas las primeras víctimas de esta revolución? Revista de Ingeniería y Gestión Industrial, 8(5), 1512-1532.

Spence, C., Chabrak, N. \& Pucci, R. (2013). Doxic sunglasses: A response to "Green accounting and Green Eyeshades: Twenty years later”. Critical Perspectives on Accounting. September 24(6): 469-473. https://doi.org/10.1016/j.cpa.2013.05.002

Taleb, M. (2015). Fifty years of Sustainability Accounting: does accounting for income in business sustainability really exist? International Journal of Accounting and Financial Reporting, 5(1), 36-47. 
Urraca, E., y Silva, J. (2017). Diagnóstico, evaluación y propuesta de manejo ambiental de los residuos sólidos y efluentes en una industria panificadora periodo junio-julio 2015. Revista ciencia y tecnología, 12(3), 25-39.

Welford, R. (2016). Corporate environmental management 1: Systems and strategies. Routledge: Florida.

Wójcik, D. (2015). Accounting for globalization: evaluating the potential effectiveness of country-by-country reporting. Environment and Planning C: Government and Policy, 33(5), 1173-1189.

Van Thanh, N., Chamorro, C., Hens, L., \& Lan, T. (2016). The widening concept of "cleaner production”. Cultura Educación y Sociedad, 7(2), 9-25. 